Table 1. Summary of Positive Predictive Values (PPV) applying our algorithm to identify AAV diagnoses and RTX use

\begin{tabular}{|c|c|c|c|}
\hline & Trust 1 & Trust 2 & Combined \\
\hline $\begin{array}{l}\text { Diagnosis of AAV and coded as } \\
\text { AAV }\end{array}$ & 69 & 44 & 113 \\
\hline AAV coded & 74 & 46 & 120 \\
\hline Diagnosis of AAV under any code & 73 & 55 & 128 \\
\hline PPV AAV ascertainment $(95 \% \mathrm{Cl})$ & $\begin{array}{c}93.2 \% \\
(84.9-97.8)\end{array}$ & $\begin{array}{c}95.7 \% \\
(85.2-99.5)\end{array}$ & $\begin{array}{c}94.2 \% \\
(88.4-97.6)\end{array}$ \\
\hline $\begin{array}{l}\text { Sensitivity of AAV ascertainment } \\
\qquad(95 \% \mathrm{Cl})\end{array}$ & $\begin{array}{c}94.5 \% \\
(86.8-98.5)\end{array}$ & $\begin{array}{c}80.0 \% \\
(67.0-89.6)\end{array}$ & $\begin{array}{c}88.3 \% \\
(81.4-93.3)\end{array}$ \\
\hline RTX given in people coded as AAV & 56 & 15 & 71 \\
\hline RTX coded in people coded as AAV & 59 & 17 & 76 \\
\hline $\begin{array}{l}\text { RTX given for AAV under any } \\
\text { diagnostic or procedure code }\end{array}$ & 64 & 23 & 87 \\
\hline PPV RTX ascertainment $(95 \% \mathrm{Cl})$ & $\begin{array}{c}94.9 \% \\
(85.9-98.9)\end{array}$ & $\begin{array}{c}88.2 \% \\
(63.6-98.5)\end{array}$ & $\begin{array}{c}93.4 \% \\
(85.3-97.8)\end{array}$ \\
\hline $\begin{array}{l}\text { Sensitivity of RTX ascertainment } \\
\qquad(95 \% \mathrm{Cl})\end{array}$ & $\begin{array}{c}87.5 \% \\
(76.8-94.4)\end{array}$ & $\begin{array}{c}65.2 \% \\
(42.7-83.6)\end{array}$ & $\begin{array}{c}81.6 \% \\
(71.9-89.1)\end{array}$ \\
\hline
\end{tabular}

Conclusion: HES data identified patients treated with RTX for AAV with a PPV of $93.4 \%$ (85.3-97.8) and sensitivity of $81.6 \%$ (71.9-89.1). This demonstrates the utility of national data to identify people receiving RTX for AAV. The RECORDER project, within the National Disease Registration Service plans to conduct realworld studies of the high-cost drug RTX, given for AAV, across the whole of England, and assess whether geography, demographics or socioeconomic factors influence frequency of prescription of this, and potentially other, high-cost drugs in line with the NHS long term plan.

REFERENCES:

[1] Ward-Platt M, Stevens S, Miller N. I18 The national congenital anomaly and rare disease registration service (NCARDRS): The first year

Acknowledgements: I have no acknowledgements to declare.

Disclosure of Interests: Cattleya Godsave: None declared, Mithun Chakravorty: None declared, Megan Rutter: None declared, Peter Lanyon Grant/research support from: P.C.L. is a recipients of a grant from Vifor Pharma. Vifor Pharma had no influence on the design, conduct or interpretation of this study., Jeanette Aston: None declared, Mary Bythell: None declared, Sarah Stevens: None declared, Fiona Pearce Grant/research support from: I have received a research grant from Vifor Pharma

DOI: 10.1136/annrheumdis-2021-eular.1591

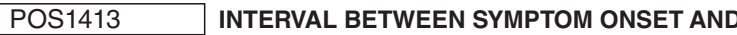 DIAGNOSIS AMONG PATIENTS WITH AUTOIMMUNE RHEUMATIC DISEASES IN A MULTI-ETHNIC ASIAN POPULATION}

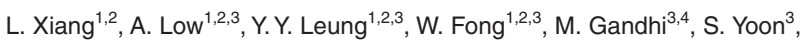
T. C. Lau ${ }^{2,5}$, D. R. Koh ${ }^{2,5}$, J. Thumboo ${ }^{1,2,3} .{ }^{1}$ Singapore General Hospital, Department of Rheumatology and Immunology, Singapore, Singapore; ${ }^{2}$ National University of Singapore, Yong Loo Lin School of Medicine, Singapore, Singapore; ${ }^{3}$ Duke-NUS Medical School, NA, Singapore, Singapore; ${ }^{4}$ Singapore Clinical Research Institute, Biostatistics, Singapore, Singapore; ${ }^{5}$ National University Hospital, Department of Medicine, Singapore, Singapore

Background: The interval between symptom onset and diagnosis can often be longer than is ideal in autoimmune rheumatic diseases (ARDs).

Objectives: We aimed to characterise this interval among patients newly diagnosed with ARDs in a multi-ethnic Asian population and to identify factors associated with a longer interval.

Methods: We used Scott's model of pathways to treatment to characterise the interval between symptom onset and diagnosis into 4 intervals: \#1 between symptom onset and first seeking medical attention, \#2 between first medical attention and rheumatology referral, \#3 between rheumatology referral and first rheumatology assessment, and \#4 between first rheumatology assessment and diagnosis. Linear regression models were used to identify factors associated with a longer the overall interval between symptom onset and diagnosis and Interval $\# 1$.

Results: Among 259 patients (age: $51 \pm 15$ years, female: $71 \%$, most common three ARDs: rheumatoid arthritis $(n=75)$, axial spondyloarthritis $(n=40)$ and psoriatic arthritis $(n=35)$ ), the median overall interval was 11.5 months. Interval \#1 (median = 4.9 months) was significantly longer than the other intervals (Table 1). Patients with axial spondyloarthritis had a significantly longer overall interval (median $=38.7$ months) and Interval \#1 (median = 26.6 months) compared to patients with RA (median $=7.6$ and 3.5 months, respectively), PsA (median $=7.0$ and 2.6 months, respectively) and the other ARDs. Gender was the only patient-related factor significantly associated with the overall interval (reference $=$ male, coefficient $=-15.3, p=0.033$ ) in regression models.
Conclusion: A longer than ideal interval between symptom onset and diagnosis was observed among patients with ARDs. This was primarily due to a relatively long interval between symptom onset and first seeking medical attention, and highlights the importance of interventions targeting patients prior to first medical attention in reducing the duration between symptom onset and diagnosis.

REFERENCES:

[1] Scott SE, Walter FM, Webster A, Sutton S, Emery J. The model of pathways to treatment: conceptualization and integration with existing theory. $\mathrm{Br}$ J Health Psychol. 2013;18(1):45-65.

Table 1. Interval between symptom onset and diagnosis

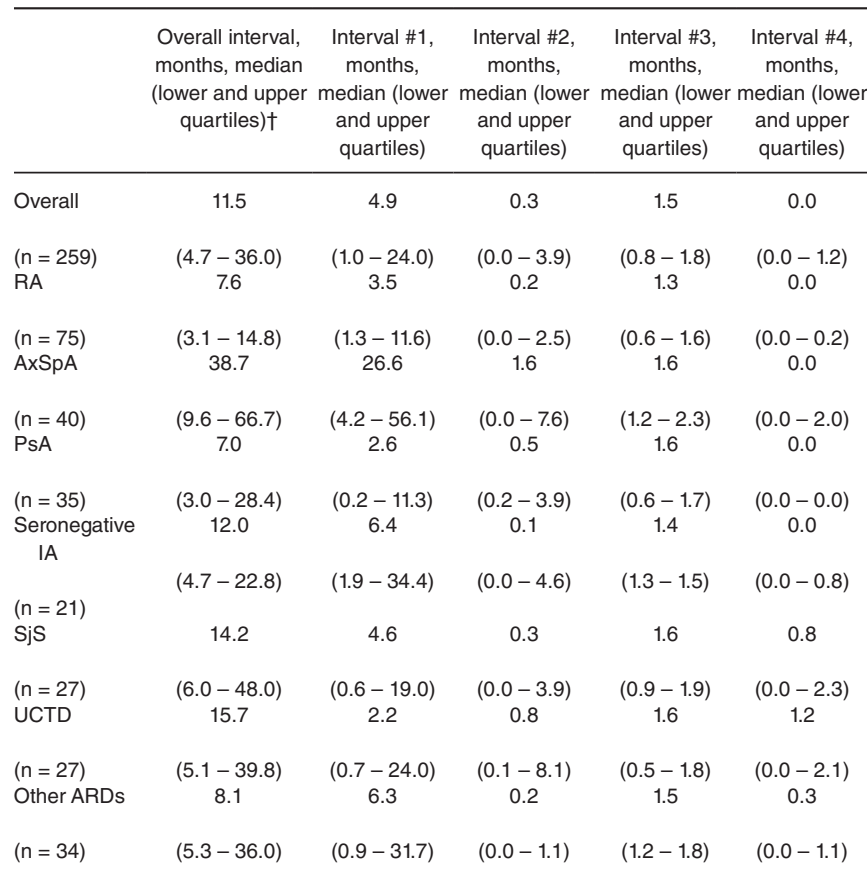

Overall interval and Intervals \#1-4: refer to abstract for definitions; RA: rheumatoid arthritis; axSpA: axial spondyloarthritis; PsA: psoriatic arthritis; IA: inflammatory arthritis; SjS: Sjögren's syndrome; UCTD: undifferentiated connective tissue disease; other ARDs: systemic lupus erythematosus, systemic sclerosis, idiopathic inflammatory myopathies, palindromic rheumatism and overlap syndromes. fIntervals \#1-4 did not sum to the overall interval mainly due to the fact that Intervals \#1-4 might not available for all patients.

Disclosure of Interests: None declared

DOI: 10.1136/annrheumdis-2021-eular.1713

\section{POS1414 RHEUMATOID ARTHRITIS AND INTERSTITIAL LUNG DISEASE: PREVALENCE AND DRUG PRESCRIPTIONS IN GERMAN CLAIMS DATA}

K. Albrecht ${ }^{1}$, A. Strangfeld ${ }^{1}$, U. Marschall ${ }^{2}$, J. Callhoff ${ }^{1} .{ }^{1}$ German Rheumatism Research Centre, Epidemiology, Berlin, Germany; ${ }^{2} B A R M E R$ Institute for Health System Research, Department Medicine and Health Services Research, Berlin, Germany

Background: Persons with rheumatoid arthritis (RA) have an increased risk of interstitial lung disease (ILD). ILD is a serious extraarticular manifestation in RA with a significantly increased mortality but without evidence-based drug therapy (1)

Objectives: The aim of this analysis was to investigate the frequency of ILD diagnosis in RA using claims data and to identify the medications prescribed. Methods: Data from a large German statutory health insurance fund were used to identify persons with one inpatient or two outpatient diagnoses of RA (ICD-10: M05, M06) and ILD (J84.1, J84.8, J84.9 and M05.1+J99.0) in 2019. Specialist care by rheumatologists and/or pulmonologists was identified using physician specialty numbers. Drug prescriptions of glucocorticoids, conventional synthetic disease modifying antirheumatic drugs (csDMARDs: methotrexate, leflunomide, sulfasalazine, hydroxychloroquine, mycophenolate), biologic (b) DMARDs (abatacept, rituximab, TNF inhibitors, tocilizumab) or targeted synthetic (ts) DMARDs (tofacitinib) were identified by ATC codes. Prescriptions were included if a person received at least one prescription of the respective drug in 2019.

Results: Among 7,479,000 persons over 18 years of age and insured in 2019 a total of $2.0 \%(n=148,000)$ had a diagnosis of RA and $1.1 \%(n=1,600)$ of those had an additional diagnosis of ILD. The majority of persons with RA+ILD 
diagnosis was older than 70 years (59\%), mean age was 72 years, $68 \%$ were female and $41 \%$ had a diagnosis of serpositive RA (M05). 4 out of 5 patients were in rheumatologist or pulmonologist care (36\% both, $22 \%$ only rheumatologist, $22 \%$ only pulmonologist). In total, $67 \%$ received glucocorticoids, $49 \%$ csDMARDs and $19 \%$ bDMARDs and $1.8 \%$ tofacitinib. TNF inhibitors were the most frequently prescribed bDMARDs followed by abatacept and tocilizumab. Persons without specialized care received considerably less DMARD therapy (Table 1). Nintedanib was prescribed to 14 patients, pirfenidon to 10 patients, all of them were in specialist care.

Table 1. Demographics and treatment of persons with RA and ILD diagnosis, numbers are percentages unless indicated otherwise.

\begin{tabular}{lccc}
\hline Variable & $\begin{array}{c}\text { Not treated by } \\
\text { rheumatologist or } \\
\text { pulmonologist }\end{array}$ & $\begin{array}{c}\text { Treated by } \\
\text { rheumatologist and/or } \\
\text { pulmonologist }\end{array}$ & All \\
\hline $\mathrm{N}(\%)$ & $326(20 \%)$ & $1274(80 \%)$ & $1,600(100 \%)$ \\
Age, mean years (std) & $75(10)$ & $72(10)$ & $72(10)$ \\
$<70$ years & 35 & 43 & 41 \\
70 to 80 years & 31 & 39 & 37 \\
$>80$ years & 35 & 18 & 22 \\
Female sex & 67 & 69 & 68 \\
Glucocorticoids & 49 & 72 & 67 \\
csDMARDs & 24 & 56 & 49 \\
MTX & 13 & 30 & 27 \\
Leflunomide & 4.9 & 10 & 8.9 \\
(Hydroxy-)chloroquine & 2.8 & 9.0 & 7.8 \\
Mycophenolate & 1.2 & 2.2 & 2.0 \\
Sulfasalazine & 2.1 & 3.8 & 3.4 \\
bDMARDs & 7 & 22 & 19 \\
TNF-alpha Inhibitors & 3.4 & 9.0 & 7.9 \\
Abatacept & 2.1 & 5.6 & 4.9 \\
Tocilizumab & 1.2 & 3.6 & 3.1 \\
Rituximab & 0.9 & 2.9 & 2.5 \\
Tofacitinib & 1.5 & 1.9 & 1.8 \\
\hline
\end{tabular}

Conclusion: ILD was diagnosed in one of 100 persons with RA diagnosis. Specialist care is necessary to provide disease-specific therapies. While methotrexate is the most commonly used DMARD, the bDMARD prescription is heterogeneous.

REFERENCES:

[1] Hyldgaard C, Ellingsen T, Hilberg O, Bendstrup E. Rheumatoid Arthritis-Associated Interstitial Lung Disease: Clinical Characteristics and Predictors of Mortality. Respiration. 2019;98(5):455-460.

Acknowledgements: The study was supported by the German Federal Ministry of Education and Research within the network TARISMA [01EC1902A].

Disclosure of Interests: Katinka Albrecht: None declared, Anja Strangfeld: None declared, Ursula Marschall Employee of: Employee of the BARMER statutory health insurance fund, Johanna Callhoff: None declared DOI: 10.1136/annrheumdis-2021-eular.2355

\section{POS1415 SOCIO-ECONOMIC IMPACT OF SJÖGREN'S SYNDROME IN WESTERN SWITZERLAND: A CROSS- SECTIONAL STUDY}

K. Cunha Rodrigues ${ }^{1}$, A. Grosjean ${ }^{2}$, T. Hügle ${ }^{1}$, A. Dumusc ${ }^{1} .{ }^{1}$ University Hospital Lausanne, Rheumatology, Lausanne, Switzerland; ${ }^{2}$ Sjögren's Patients Association for Western Switzerland, (ARSYS), Vevey, Switzerland

Background: Several studies investigated the socio-economic impact of Sjögren's syndrome (SS) showing an increased risk of work disability and a reduced health-related quality of life. SS is also associated with increased direct and indirect healthcare costs. There are no published data about Switzerland, a country with high resources and easy access to healthcare except dental care, which is rarely reimbursed.

Objectives: To evaluate the professional and economic impact of SS in patients living in Western Switzerland.

Methods: Cross-sectional survey-based study conducted in December 2020, during the pandemic. Patients suffering from SS and living in Western Switzerland (Geneva and Lausanne region) were invited by the regional association of patients with SS to complete a survey assessing the socio-economic impact of SS.

Results: Among the 86 patients who were contacted, 55 (64\%) completed the survey. Participants were predominantly female (95\%) with a mean (SD) age of 54.2 (12.3). Mean (SD) EULAR SS Patient Reported Index (ESSPRI) was 6.5 (1.6), assessing pain, fatigue and dryness.

In the working age population (under $65, \mathrm{n}=39$ ), $64 \%$ of the participants reported to be employed, $88 \%$ of whom working part-time. They reported to work 23.3 (10.1) hours per week (mean, SD). 60\% reported SS-related work incapacity periods during the past year. $72 \%$ of participants had to reduce their working hours and $27 \%$ had to change careers due to their medical condition. $16 \%$ benefited from an adaptation of their workstation to their health status.

In terms of work disability, $27 \%$ of the participants depended on disability insurance pension, of whom $38 \%$ received a full pension. $16 \%$ of the participants applied for a disability pension and were waiting for an administrative decision. $A$ minority of participants (11\%) reported to receive a minimum subsistence allowance from the local social service.

Participants estimated to pay 2752 CHF (3000) per year (median, IQR) out of their own pocket (Swiss monthly median wage: $6500 \mathrm{CHF}$ ) for health care not covered by health insurances. $95 \%$ of the patients had to pay for dental medicine costs, at least partially. Public health insurance contributed to dental costs for $44 \%$ of the patients and private health insurance for $28 \%$ of them. $22 \%$ of the patients reported to limit dental care for financial reasons.

Conclusion: Two-thirds of the patients with SS remained in active employment, but the majority of them working in part-time, with a substantial loss of income. One third of the patients are work disabled and depend on a disability pension. Dental care was not reimbursed in half of the patients by their health insurance, leading to dental care access restriction for $20 \%$ of the patients with SS. Despite a relatively high wage level in Switzerland, SS represents a substantial financial burden for most of the patients.

\section{REFERENCES:}

[1] Mandl T, Jørgensen TS, Skougaard M, Olsson P, Kristensen LE. Work Disability in Newly Diagnosed Patients with Primary Sjögren Syndrome. J Rheumatol. 2017 Feb;44(2):209-215

[2] Meijer JM, Meiners PM, Huddleston Slater JJ, Spijkervet FK, Kallenberg CG, Vissink A, Bootsma H. Health-related quality of life, employment and disability in patients with Sjogren's syndrome. Rheumatology. 2009 Sep;48(9):1077-82.

[3] Westhoff G, Dörner T, Zink A. Fatigue and depression predict physician visits and work disability in women with primary Sjögren's syndrome: results from a cohort study. Rheumatology. 2012 Feb;51(2):262-9.

[4] Miyamoto ST, Valim V, Fisher BA. Health-related quality of life and costs in Sjögren's syndrome. Rheumatology. 2019 Feb 15:key370. doi: 10.1093/rheumatology/key370. Epub ahead of print.

Disclosure of Interests: None declared

DOI: 10.1136/annrheumdis-2021-eular.2721

\section{POS1416 THE IMPACT OF NON-PERSISTENCE ON RESOURCE UTILIZATION COSTS IN IMMUNE-MEDIATED RHEUMATIC DISEASES}

N. Carballo ${ }^{1}$, E. Garcia-Alzorriz Morral ${ }^{2}$, O. Ferrández-Quirante ${ }^{1}$, C. PerezGarcia $^{3}$, M. E. Navarrete-Rouco ${ }^{1}$, X. Duran ${ }^{4}$, J. Monfort ${ }^{3}$, F. Cots ${ }^{2}$, S. Grau ${ }^{1}$. ${ }^{1}$ Hospital del Mar, Pharmacy Department, Barcelona, Spain; ${ }^{2}$ Hospital del Mar, Management Control Department, Barcelona, Spain; ${ }^{3} \mathrm{Hospital}$ del Mar, Rheumatology Department, Barcelona, Spain; ${ }^{4}$ Institute Hospital del Mar for Medical Research (IMIM), Methodology and Biostatistics Support Unit, Barcelona, Spain

Background: Rheumatoid arthritis (RA), psoriatic arthritis (PsA) and ankylosing spondylitis (AS) are chronic progressive immune-mediated rheumatic diseases (IMRD) that can cause a progressive disability and joint deformation and thus can impact in healthcare resource utilization (HCRU) and costs.

Objectives: To describe the HCRU and treatment costs in IMRD patients initiating subcutaneous tumour necrosis factor-alpha inhibitors (SC-TNFi) therapy, based on treatment persistence.

Methods: Retrospective cohort study including all naïve patients initiating SC-TNFi therapy for IMRD from 2015-2018 in a tertiary university hospital. Patients were divided into two cohorts: persistent and non-persistent. Treatment persistence was estimated as the duration of time from SC-TNFi therapy initiation to discontinuation during one year of follow-up.

SC-TNFi therapy and HCRU costs (outpatient care, rheumatology specialized outpatient care, inpatient care, emergency care, laboratory testing and other non- biological therapies) were calculated one year before and after initiation of SC-TNFi and compared between persistence and non-persistence groups.

Results: 110 patients were identified.

Baseline characteristics: Non-persistent cohort $(n=25)$ versus Persistent cohort $(n=85)$ : median age $48.6(12.7)$ vs $47.3(15.4) \quad(p=0.692)$. Female $(n=12 ; 48 \%)$ vs $(n=49 ; 57.6 \%) \quad(p=0.493)$. Race: Caucasian $(n=22 ; 88 \%)$, Asiatic $(n=3 ; 12 \%)$, Other $(n=0 ; 0 \%)$ vs Caucasian $(n=75 ; 88.2 \%)$, Asiatic $(n=5 ; 5.9 \%)$, Other $(n=5 ; 5.9 \%) \quad(p=0.351)$.IMRD: RA $(n=14 ; 56 \%), \operatorname{PsA}(n=2 ; 8 \%)$, AS $(n=4 ; 16 \%)$, other spondyloarthropathy $(n=5 ; 20 \%)$ vs RA ( $n=34 ; 40 \%)$,PsA $(n=11 ; 12.9 \%)$, AS $(n=24 ; 28.3 \%)$, other spondyloarthropathy $(n=16 ; 18.8 \%)(p=0.470)$. SC-TNFi therapy: adalimumab $(n=4 ; 16 \%)$, etanercept commercial $(n=4 ; 16 \%)$, etanercept biosimilar1 ( $n=5 ; 20 \%)$, etanercept biosimilar2 $(n=5 ; 20 \%)$, golimumab $(n=5 ; 20 \%)$, certolizumab $(n=2 ; 8 \%)$ vs adalimumab $(n=22 ; 25.9 \%)$, etanercept commer cial $(n=11 ; 12.9 \%)$, etanercept biosimilar1 ( $n=7 ; 8.3 \%)$, etanercept biosimilar2 $(n=10 ; 11.8 \%)$, golimumab $(n=24 ; 28.2 \%)$, certolizumab $(n=11 ; 12.9 \%)(p=0.398)$. 